\title{
Minimizing within convex bodies using a convex hull method
}

\author{
Thomas Lachand-Robert Édouard Oudet*
}

11th May 2004

\begin{abstract}
We present numerical methods to solve optimization problems on the space of convex functions or among convex bodies. Hence convexity is a constraint on the admissible objects, whereas the functionals are not required to be convex. To deal with, our method mix geometrical and numerical algorithms.

We give several applications arising from classical problems in geometry and analysis: Alexandrov's problem of finding a convex body of prescribed surface function; Cheeger's problem of a subdomain minimizing the ratio surface area on volume; Newton's problem of the body of minimal resistance.

In particular for the latter application, the minimizers are still unknown, except in some particular classes. We give approximate solutions better than the theoretical known ones, hence demonstrating that the minimizers do not belong to these classes.
\end{abstract}

Keywords: Optimization, Convex functions, Numerical schemes, Convex bodies, Newton's problem of the body of minimal resistance, Alexandrov, Cheeger.

AMS classification: 46N10, 52A40, 52A41.

\footnotetext{
*Laboratoire de mathématiques, Université de Savoie, Campus scientifique, 73376 Le Bourget-du-lac, France. Thomas.Lachand-Robert@univ-savoie.fr, Edouard.Oudet@univ-savoie.fr http://www.lama.univ-savoie.fr/ lachand, http://www.lama.univ-savoie.fr/ oudet.
} 


\section{Introduction}

In this paper, we present numerical methods to solve optimization problems among convex bodies or convex functions. Several problems of this kind appear in geometry, calculus, applied mathematics, etc. As applications, we present some of them together with our corresponding numerical results.

Dealing with convex bodies or convex functions is usually considered easier in optimization theory. Unfortunately this is not true when the optimization space itself is (a subset of) the set of convex functions or bodies. As an example, consider the following minimization problem, where $M>0$ is a given parameter, $\Omega$ a regular bounded convex subset of $\mathbb{R}^{n}$ and $g$ a continuous function on $\Omega \times \mathbb{R} \times \mathbb{R}^{n}$ :

$$
\begin{gathered}
\inf _{u \in C_{M}} \int_{\Omega} g(x, u(x), \nabla u(x)) d x, \\
\text { where } C_{M}=\{u: \Omega \rightarrow[-M, 0], \quad u \text { convex }\} .
\end{gathered}
$$

Without the convexity constraint, this problem is usually handled in a numerical way by considering the associated Euler equation $g_{2}^{\prime}(x, u(x), \nabla u(x))=$ $\left.\operatorname{div} g_{3}^{\prime}(x, u(x), \nabla u(x))\right)$. Such an equation is discretized and solved on a mesh defined on $\Omega$ (or more precisely, a sequence of meshes, in order to achieve a given precision), using for instance finite element methods.

\subsection{Dealing with the convexity constraint}

The classical numerical methods do not work at all with our problem:

1. The convexity constraint prevents us from using an Euler equation. In fact, just stating a correct Euler equation for this sort of problem is a difficult task $[12,20,8]$. Discretizing the corresponding equation is rather difficult, then.

2. The set $C_{M}$ of admissible functions, considered as a subset of a Sobolev space like $H_{\text {loc }}^{1}(\Omega)$, is compact [5]. This makes it easy to prove the existence of a solution of (1) without any other assumption on $g$. But this also implies that $C_{M}$ is a very small subset of the functions space, with empty interior. Therefore most numerical approximations of a candidate function $u$ are not convex. Evaluating the functional on those approximations is likely to yield a value much smaller than the sought minimum.

3. The natural way to evade the previous difficulty is to use only convex approximations. For instance, on a triangular mesh of $\Omega$, it is rather 
easy to characterize those P1-functions (that is, continuous and affine by parts functions) which are convex. Unfortunately, such an approximation introduces a geometric bias from the mesh. The set of convex functions that are limits of this sort of approximation is much smaller than $C_{M}[13]$.

4. Penalization processes are other ways to deal with this difficulty. But finding a good penalization is not easy, and this usually yields very slow algorithms, which in this particular case are not very convincing. This yields approximation difficulties similar to those given in 2 above.

A first solution for this kind of numerical problems was presented in [10], and an improved version is given in [9]. However the algorithms given in these references are not very fast, since they deal with a large number of constraints, and do not apply to those problems where local minimizers exist. The latter are common in the applications since there is not need for the functional itself to be convex to prove the existence of solution of (1): the mere compacity of $C_{M}$, together with the continuity of the functional on an appropriate functions space, is sufficient.

\subsection{A mixed-type algorithm}

Our main idea to handle numerically (1) is to mix geometrical and numerical algorithms. It is standard that any convex body (or equivalently, the graph of any convex function) can be described as an intersection of half-spaces or as a convex hull of points. Our discretization consists in considering only a finite number of half-spaces, or a finite number of points (this is not equivalent, and choosing either mode is part of the method). Reconstructing the convex body is a standard algorithm, and computing the value of the functional is straightforward then. Obviously the convex hull algorithm used implies an additional cost that can not be neglected. On the other hand, this method makes it easy to deal with additional constraints like the fact that functions get values in $[0, M]$, for instance. We also show that it is possible to compute the derivative of the functional. Hence we may use gradient methods for minimization.

Note that since this always deals with convex bodies, we are guaranteed that the evaluations of the functional are not smaller than the sought minimum, up to numerical errors. Because the approximation process is valid for any convex body, we can ensure that all minimizers can be approximated arbitrary closely.

The detailed presentation of the method requires to explain how the halfspaces or points are moved, whether or not their number is increased, and 
which information on the specific problem is useful for this. We present quite different examples in our applications, in order to pinpoint the corresponding difficulties. Whenever the minimizer of the functional is not unique, gradient methods may get stuck in local minima. We present a "genetic algorithm" to deal with these, too.

In this paper, we concentrate on the three-dimensional settings. The two-dimensional case is much easier, and convex sets in the plane can be parametrized in a number of very simple ways. Even though our methods could be applied to dimensions $n \geq 4$, the convex hull computation may become too expensive.

\subsection{Generalized problem}

This algorithm's design does not involve any mesh or interpolation process. As an important consequence, we are not limited to convex functions but may also consider convex bodies. This allows us to study problems like

$$
\inf _{A \in \mathcal{A}} \mathcal{F}(A), \quad \text { where } \mathcal{F}(A):=\int_{\partial A} f\left(x, \nu_{A}(x), \varphi_{A}(x)\right) d \mathcal{H}^{2}(x),
$$

and $\mathcal{A}$ is a subset of the class of closed convex bodies of $\mathbb{R}^{3}$. We make use of the notations:

- $\partial A$ is the boundary of a convex body $A$;

- $\nu_{A}$ is the almost everywhere defined outer normal vector field on $\partial A$, with values on the sphere $\mathbf{S}^{2}$;

- $\varphi_{A}(x)$ is the signed distance from the supporting plane at $x$ to the origin of coordinates;

- $f$ is a continuous function $\mathbb{R}^{3} \times \mathbf{S}^{2} \times \mathbb{R} \rightarrow \mathbb{R}$.

Since $\varphi_{A}(x)=x \cdot \nu_{A}(x)$ the expression of the functional $\mathcal{F}$ is somehow redundant. But the particular case of functions $f$ depending only on $\nu, \varphi$ is important both in applications and in the algorithm used, as we shall see.

As reported in [7], the problem (1) can be reformulated in terms of (2) whenever $g$ depends only on its third variable. In this formulation $\mathcal{A}$ stands for the set of convex subsets of $Q_{M}:=\Omega \times[0, M]$ containing $Q_{0}=\Omega \times\{0\}$. Any convex body $A \in \mathcal{A}$ has the form

$$
\mathcal{A}=\left\{\left(x^{\prime}, x_{3}\right) \in \Omega \times \mathbb{R}, 0 \leq x_{3} \leq-u\left(x^{\prime}\right)\right\} \text {, with } u \in C_{M} .
$$


Therefore any $x \in \partial A \backslash Q_{0}$ has the form $x=\left(x^{\prime},-u\left(x^{\prime}\right)\right)$, with $x^{\prime} \in \Omega$. Then $\nu_{A}(x)=\left(\nabla u\left(x^{\prime}\right), 1\right) / \sqrt{1+\left|\nabla u\left(x^{\prime}\right)\right|^{2}}$, and the function $f$ is deduced from $g$ by the relation $f(\nu)=\nu_{3} g\left(\frac{1}{\nu_{3}} \nu^{\prime}\right)$, for every $\nu=\left(\nu^{\prime}, \nu_{3}\right) \in \mathbf{S}^{2}$. Several other problems with a geometrical background may also be formulated in a similar way.

Actually the formulation (2) allows us to study any problem of the form (1). It is enough to define $f(x, \nu, \varphi)=\nu_{3} g\left(x^{\prime},-x_{3}, \frac{1}{\nu_{3}} \nu^{\prime}\right)$, taking into account that $x=\left(x^{\prime},-u\left(x^{\prime}\right)\right)$.

On the other hand, it is much more practical in the numerical implementation to consider functions $f$ depending only on $\nu, \varphi$. This avoids numerical surface integration altogether, as explained in section 2 hereafter, hence reducing greatly the computation time. With such a restriction, only some problems of the form (1) can be considered. Since

$$
\varphi_{A}(x)=\frac{1}{\sqrt{1+\left|\nabla u\left(x^{\prime}\right)\right|^{2}}}\left(x^{\prime} \cdot \nabla u\left(x^{\prime}\right)+u\left(x^{\prime}\right)\right),
$$

we can handle functions $g$ depending on $\nabla u\left(x^{\prime}\right)$ and the aggregate $x^{\prime} \cdot \nabla u\left(x^{\prime}\right)+$ $u\left(x^{\prime}\right)$.

\section{Half-spaces and discretization}

For every $\nu \in \mathbf{S}^{2}$ and every $\varphi \geq 0$, let us define the half-space of $\mathbb{R}^{3}$ using the following notation:

$$
\llbracket \nu, \varphi \rrbracket:=\left\{x \in \mathbb{R}^{3}, x \cdot \nu \leq \varphi\right\} .
$$

Lemma 1 Let $A$ be a convex body of $\mathbb{R}^{3}$. Then $\forall \varepsilon>0$, there exists a convex polytope $P \supset A$ such that:

$$
|\mathcal{F}(P)-\mathcal{F}(A)| \leq \varepsilon
$$

Proof. Let us note

$$
\partial^{*} A:=\left\{a \in \partial A ; \nu_{A}(a) \text { exists }\right\} .
$$

Let $\left(X_{j}\right)_{j \in \mathbb{N}}$ be a dense sequence of points in $\partial^{*} A$ and consider the sequence of convex polytopes $\left(P_{j}\right)_{j \in \mathbb{N}}$ defined by:

$$
P_{j}:=\bigcup_{k=1}^{j} \llbracket \nu_{A}\left(X_{k}\right), \varphi_{A}\left(X_{k}\right) \rrbracket .
$$


Clearly $P_{j} \supset A$ and $\lim _{j \rightarrow \infty} P_{j}=A$ for the Hausdorff distance. From a classical theorem of Rockafellar [22], for any $a \in \partial^{*} A$, and any sequence $\left(p_{j}\right)$, converging to $a$, with $p_{j} \in \partial^{*} P_{j}$ for all $j$, we have $\nu_{P_{j}}\left(p_{j}\right)$ converges to $\nu_{A}(a)$. Since $\partial A \backslash \partial^{*} A$ is $\mathcal{H}^{2}$-negligible, we get $\mathcal{F}\left(P_{j}\right) \rightarrow \mathcal{F}(A)$.

As every convex polytope is the finite intersection of half-spaces, the natural discretization of (2) is the finite dimensional problem:

$$
\begin{aligned}
\min _{N, \Phi} G(N, \Phi) & \\
\text { where } N & :=\left(\nu_{1}, \ldots, \nu_{k}\right) \in\left(\mathbf{S}^{2}\right)^{k}, \Phi:=\left(\varphi_{1}, \ldots, \varphi_{k}\right) \in \mathbb{R}^{k}, \\
G(N, \Phi) & :=\int_{\partial P} f\left(x, \nu_{P}(x), \varphi_{P}(x)\right) d \mathcal{H}^{2}(x), \\
\text { and } P & :=P(N, \Phi):=\bigcap_{i=1}^{k} \llbracket \nu_{i}, \varphi_{i} \rrbracket .
\end{aligned}
$$

Notice that, whenever $f$ does not depend explicitly on $x, G(N, \Phi)$ can be computed as a finite sum, namely

$$
G(N, \Phi)=\sum_{i=1}^{k} f\left(\nu_{i}, \varphi_{i}\right) \mathcal{H}^{2}\left(F_{i}\right), \text { where } F_{i}:=\llbracket \nu_{i}, \varphi_{i} \rrbracket \cap \partial P .
$$

This is of primary importance in the numerical algorithms. More general functions $f$ require the computation of integrals like $\int_{F_{i}} f\left(x, \nu_{i}, \varphi_{i}\right) d \mathcal{H}^{2}(x)$, which are computationally expensive.

\subsection{Computation of the derivatives}

In this paragraph we compute the derivatives of $G$, in order to use the results in a gradient-like method. We focus on the case where $f$ depends only on $\nu, \varphi$, since this is the special case used in our actual programs. Straightforward modifications can be done to handle the general case. It suffices to change the term $\frac{\partial f}{\partial \varphi_{i}}\left(\nu_{i}, \varphi_{i}\right) \mathcal{H}^{2}\left(F_{i}\right)$ by the integral $\int_{F_{i}} \frac{\partial f}{\partial \varphi_{i}}\left(x, \nu_{i}, \varphi_{i}\right) d \mathcal{H}^{2}(x)$, and similarly with the $\mathcal{H}^{1}$ term.

Theorem 1 Let $P:=P(N, \Phi)$ be a convex polytope and $F_{i}=\llbracket \nu_{i}, \varphi_{i} \rrbracket \cap \partial P$. Then for almost every value of $\varphi_{i}$ we have:

$$
\begin{aligned}
\frac{\partial G}{\partial \varphi_{i}}(N, \Phi)=\frac{\partial f}{\partial \varphi_{i}}\left(\nu_{i}, \varphi_{i}\right) & \mathcal{H}^{2}\left(F_{i}\right) \\
& +\sum_{\substack{j \neq i \\
\mathcal{H}^{1}\left(F_{i} \cap F_{j}\right) \neq 0}} \mathcal{H}^{1}\left(F_{i} \cap F_{j}\right)\left(\frac{f\left(\nu_{j}, \varphi_{j}\right)-\cos \theta_{i j} f\left(\nu_{i}, \varphi_{i}\right)}{\sin \theta_{i j}}\right),
\end{aligned}
$$




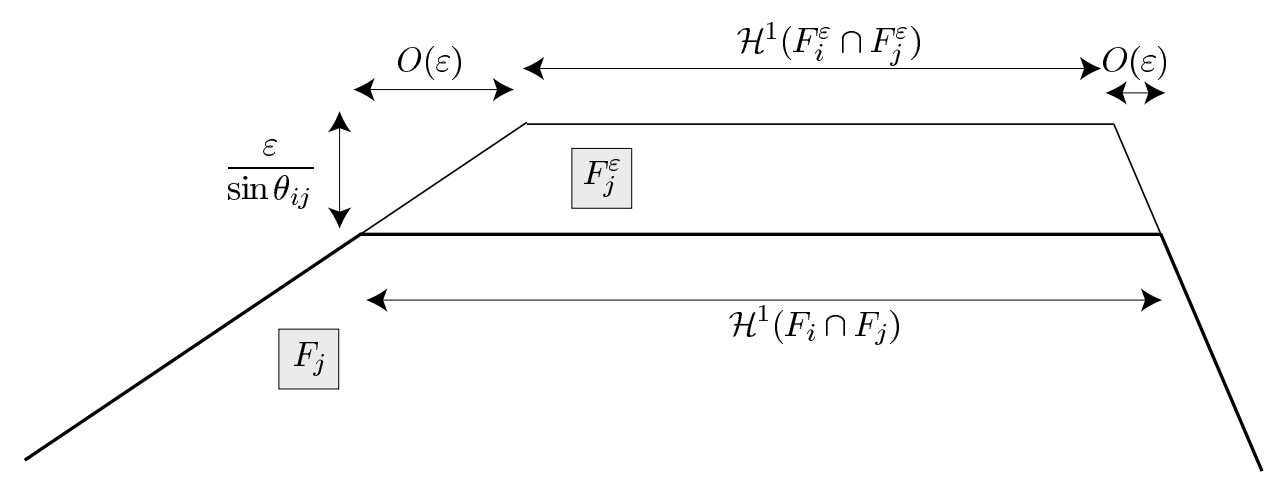

Figure 1: Variation of the surface area of $F_{j}$ (pictured in the plane of $F_{j}$ ), for the variation $\varphi_{i} \rightarrow \varphi_{i}+\varepsilon$.

where $\theta_{i j} \in\left[-\frac{\pi}{2}, \frac{\pi}{2}\right]$ is defined by $\cos \theta_{i j}=\left|\nu_{i} \cdot \nu_{j}\right|$ and $\sin \theta_{i j}\left(\nu_{i} \cdot \nu_{j}\right) \geq 0$.

Proof. For any $\varepsilon \in \mathbb{R}$ consider the difference

$$
\begin{aligned}
G\left(\ldots, \varphi_{i}+\varepsilon, \ldots\right)-G\left(\ldots, \varphi_{i}, \ldots\right) & =f\left(\nu_{i}, \varphi_{i}+\varepsilon\right) \mathcal{H}^{2}\left(F_{i}^{\varepsilon}\right)-f\left(\nu_{i}, \varphi_{i}\right) \mathcal{H}^{2}\left(F_{i}^{\varepsilon}\right) \\
& +\sum_{j} f\left(\nu_{j}, \varphi_{j}\right)\left(\mathcal{H}^{2}\left(F_{j}^{\varepsilon}\right)-\mathcal{H}^{2}\left(F_{j}\right)\right)
\end{aligned}
$$

where

$$
F_{j}^{\varepsilon}=\llbracket \nu_{j}, \varphi_{j} \rrbracket \cap \partial P\left(\ldots, \varphi_{i}+\varepsilon, \ldots\right) .
$$

The first difference $f\left(\nu_{i}, \varphi_{i}+\varepsilon\right) \mathcal{H}^{2}\left(F_{i}^{\varepsilon}\right)-f\left(\nu_{i}, \varphi_{i}\right) \mathcal{H}^{2}\left(F_{i}^{\varepsilon}\right)$ has the form $\varepsilon \frac{\partial f}{\partial \varphi_{i}}\left(\nu_{i}, \varphi_{i}\right) \mathcal{H}^{2}\left(F_{i}\right)+o(\varepsilon)$.

To evaluate the remaining sum asymptotically we have to assume that the value of $\varphi_{i}$ is such that there is no topological change in the polytope whenever $\varphi_{i}$ becomes $\varphi_{i}+\varepsilon$. This is obviously true for all except a finite number of values of $\varphi_{i}$. We then distinguish two cases:

- $j \neq i: \mathcal{H}^{2}\left(F_{j}^{\varepsilon}\right)-\mathcal{H}^{2}\left(F_{j}\right)=\varepsilon \frac{\mathcal{H}^{1}\left(F_{i} \cap F_{j}\right)}{\sin \theta_{i j}}+o(\varepsilon)$ since the trace of $F_{i}$ in the plane $F_{j}$ is offset by $\varepsilon / \sin \theta_{i j}$, see Figure 1 ;

- $j=i: \mathcal{H}^{2}\left(F_{i}^{\varepsilon}\right)-\mathcal{H}^{2}\left(F_{i}\right)=-\varepsilon \sum_{\substack{j \neq i \\ \mathcal{H}^{1}\left(F_{i} \cap F_{j}\right) \neq 0}} \mathcal{H}^{1}\left(F_{i} \cap F_{j}\right) \cot \theta_{i j}+o(\varepsilon)$, since the trace of $F_{j}$ in the plane $F_{i}$ is offset by $\varepsilon \cot \theta_{i j}$, see Figure 2 .

This completes the proof of the theorem. 


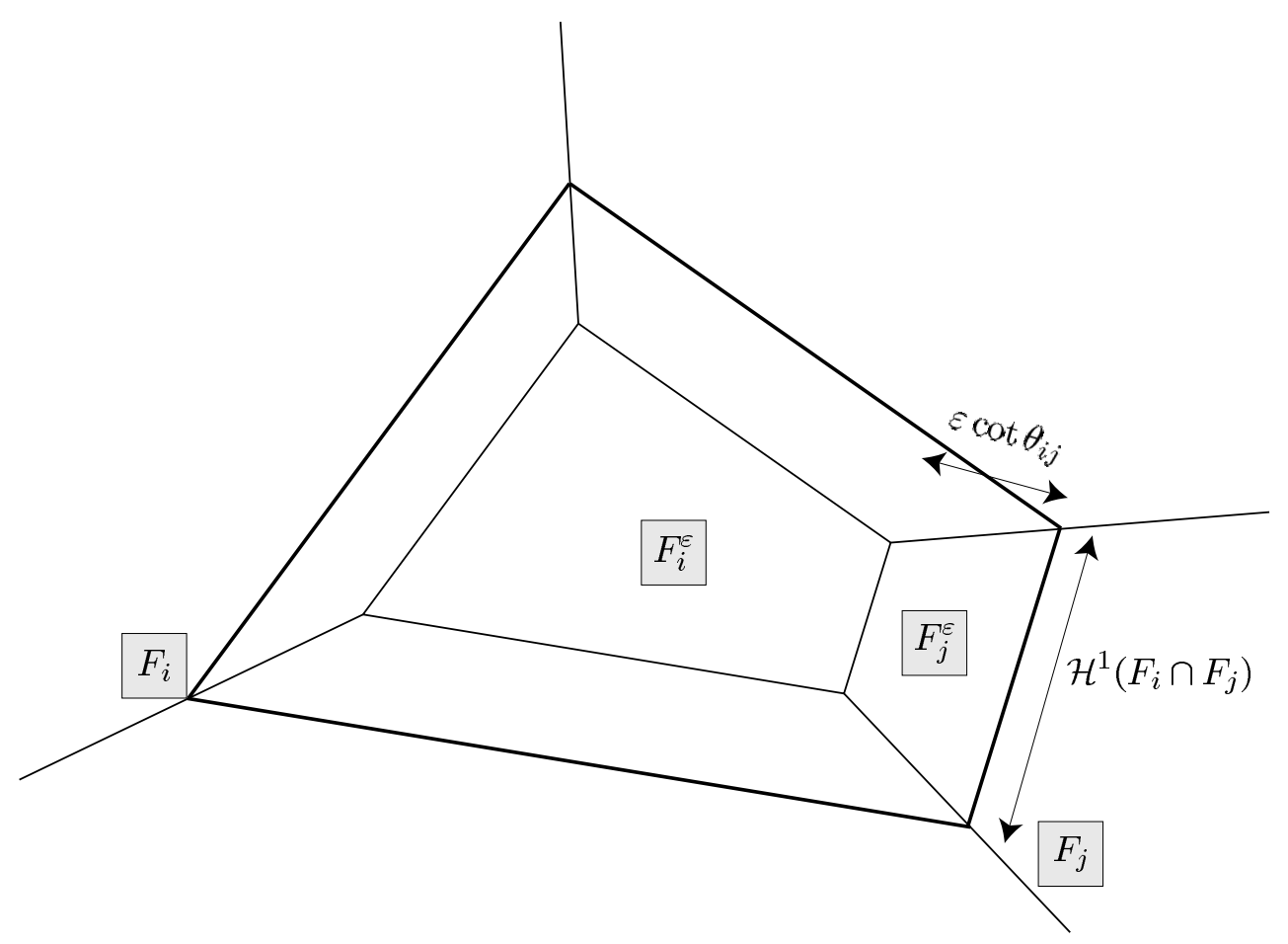

Figure 2: Variation of the surface area of $F_{i}$ (pictured in the plane of $F_{i}$ ), for the variation $\varphi_{i} \rightarrow \varphi_{i}+\varepsilon$.

Remark 2.A. The polyhedral representation, as an intersection of halfplanes, yields a technical difficulty that should not be underestimated: some of the boundary planes $\partial \llbracket \nu_{i}, \varphi_{i} \rrbracket$ are "dormant", meaning the polytope is actually included in the interior of $\llbracket \nu_{i}, \varphi_{i} \rrbracket$.

In such a situation, formula (4) effectively yields zero, since $\mathcal{H}^{2}\left(F_{i}\right)=0=$ $\mathcal{H}^{1}\left(F_{i} \cap F_{j}\right)$.

A similar computation can be achieved for derivatives of $G$ with respect to $\nu_{i}$, with another algebraic formula as a result. However numerical evidence proves that using a "full" gradient method is of little advantage.

It turns out that it is faster and accurate enough to use only the derivatives with respect to $\varphi_{i}$ (as detailed in the next section), and to increase if necessary the number of planes by considering additional half-spaces. We can make profit of the "dormant" property by introducing these new ones in a tangent dormant position, letting the minimization method changing their position after that. This can be done in different ways, depending on the 
actual problem considered.

\subsection{Summary of the algorithm}

Thanks to Theorem 1, it is possible to apply a classical gradient algorithm to the problem (3). Let us summarize the different steps:

0 . Choose one admissible polytope $P\left(\llbracket \nu_{1}, \varphi_{1}^{0} \rrbracket, \ldots, \llbracket \nu_{k}, \varphi_{k}^{0} \rrbracket\right)$, set $n=0$.

1. Compute the geometry (vertexes, faces ...) of the polytope

$$
P\left(\llbracket \nu_{1}, \varphi_{1}^{n} \rrbracket, \ldots, \llbracket \nu_{k}, \varphi_{k}^{n} \rrbracket\right) .
$$

2. Evaluate the gradient of $G$ with respect to the $\varphi_{j}$ using (4). If the Euclidean norm of the gradient is small, then stop here.

3. Project the gradient into the set of admissible directions.

4. Set $\rho_{n}=\arg \min _{\rho>0} G\left(\nu_{1}, \ldots, \nu_{k}, \varphi_{1}^{n}-\rho \frac{\partial G}{\partial \varphi_{1}}, \ldots, \varphi_{k}^{n}-\rho \frac{\partial G}{\partial \varphi_{k}}\right)$.

5. Define the new variables $\varphi_{1}^{n+1}=\varphi_{1}^{n}-\rho_{n} \frac{\partial G}{\partial \varphi_{1}}, \ldots, \varphi_{k}^{n+1}=\varphi_{k}^{n}-\rho_{n} \frac{\partial G}{\partial \varphi_{k}}$, $n \leftarrow n+1$ and go to step 1 .

Step 3 in particular depends on the set of admissible bodies. So additional details are given in the examples hereafter. Note that it is possible in step 5 to change the number of planes, by adding or removing "dormant" ones. It is also possible to change the value of $\nu_{i}$ whenever the $i$-th plane is "dormant".

\subsection{Application to Alexandrov's Theorem}

It is a classical result from Minkowski [21], that given $n$ different vectors $\nu_{1}, \ldots, \nu_{n}$ on $\mathbf{S}^{2}$ such that the dimension of $\operatorname{Span}\left\{\nu_{1}, \ldots, \nu_{n}\right\}$ is equal to 3, and $n$ positive real numbers $a_{1}, \ldots, a_{n}$ such that $\sum_{i=1}^{n} a_{i} \nu_{i}=0$, then there exists a three-dimensional convex polytope having $n$ faces $F_{1}, \ldots, F_{n}$ such that the outward normal vector to $F_{i}$ equals $\nu_{i}$ and $\mathcal{H}^{2}\left(F_{i}\right)=a_{i}$. Moreover this polytope is unique up to translations.

This result has been extended by Alexandrov [1] to arbitrary convex bodies as follows: given a positive measure $\mu$ on $\mathbf{S}^{2}$ satisfying $\int_{\mathbf{S}^{2}} y d \mu(y)=0$ and $\operatorname{Span}(\operatorname{supp} \mu)=\mathbb{R}^{3}$, then there exists a unique convex body $A$, up to translations, whose surface function measure is equal to $\mu$. 


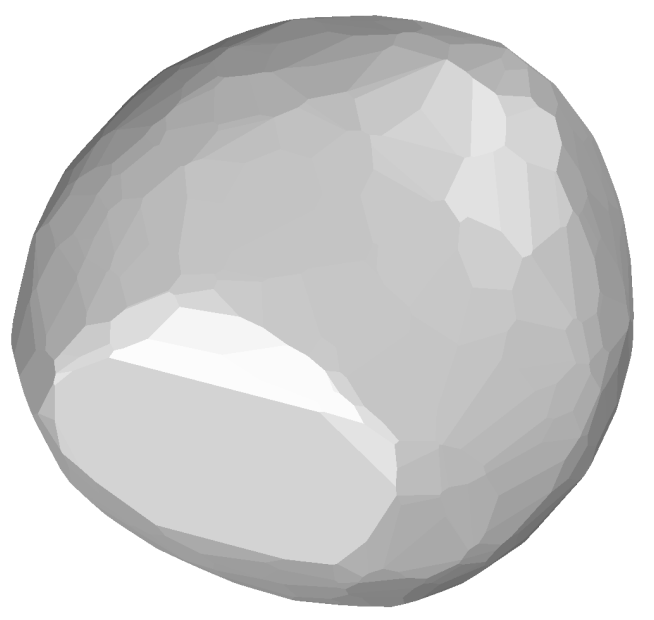

Figure 3: A 1000 faces convex polyhedron of given faces areas and normals reconstructed.

G. Carlier proved recently [6] that this body is the unique (up to translations) solution of the variational problem

$$
\sup _{\varphi \in \Sigma}\left|A_{\varphi}\right|
$$

with $\Sigma:=\left\{\varphi \in C^{0}\left(\mathbf{S}^{2}, \mathbb{R}_{+}\right) ; \int_{\mathbf{S}^{2}} \varphi d \mu=1\right\}$ and $A_{\varphi}:=\bigcap_{\nu \in \mathbf{S}^{2}} \llbracket \nu, \varphi(\nu) \rrbracket$,

where $\left|A_{\varphi}\right|$ is the volume of $A_{\varphi}$. Whenever $A_{\varphi}$ is optimal, its support function equals $\varphi$ on the support of $\mu[6]$.

Now we recall that the volume of a convex body can be expressed as a boundary integral of its support function, that is:

$$
|A|=\frac{1}{3} \int_{\partial A} \varphi_{A}(x) d \mathcal{H}^{2}(x) .
$$

Consequently Alexandrov's problem can be formulated in the form (2) with $f(x, \nu, \varphi)=-\varphi$ and

$$
\mathcal{A}=\left\{A \subset \mathbb{R}^{3}, A \text { convex } ; \varphi_{A} \geq 0, \int_{\mathbf{S}^{2}} \varphi_{A} d \mu=1\right\} .
$$

(The sign condition on $\varphi_{A}$ is only a normalization expressing the fact that $0 \in A$.)

Whenever $\mu$ has a discrete support, namely $\mu=\sum a_{i} \delta_{\nu_{i}}$, then (5) solves Minkowski's problem for polytopes. In particular, the value of $\varphi$ outside the 

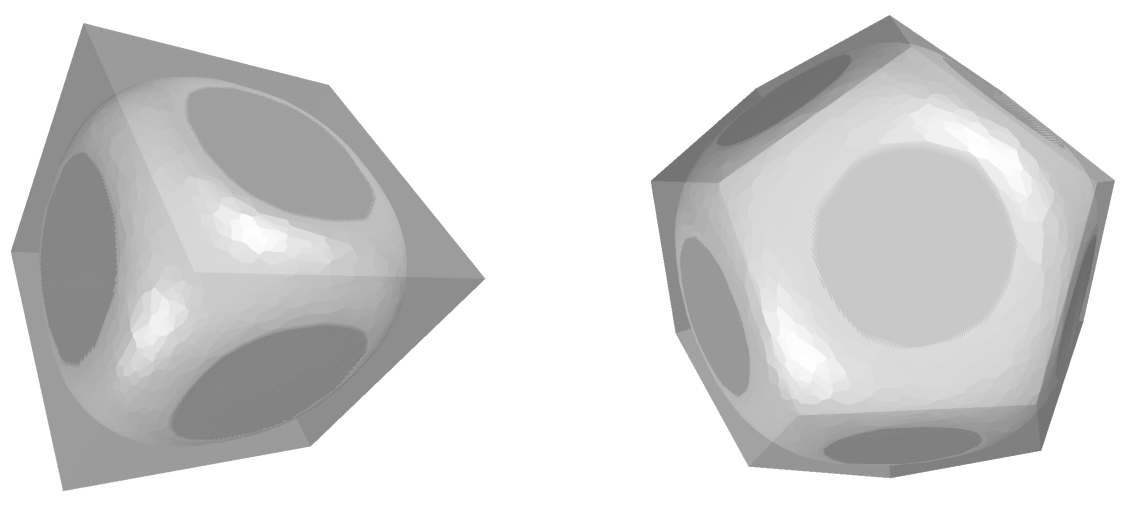

Figure 4: Computed solutions for the Cheeger problem in the cube and the dodecahedron.

support of $\mu$ does not matter for the maximization, hence only the numbers $\varphi_{i}:=\varphi\left(\nu_{i}\right)$ have to be considered.

Replacing an arbitrary measure $\mu$ on $\mathbf{S}^{2}$ by a sum of Dirac masses is also the more natural discretization of this problem. For polytopes, the set of admissible bodies has the form

$$
\mathcal{A}=\left\{P=P(N, \Phi) ; \varphi_{i} \geq 0, \sum_{i=1}^{n} \varphi_{i} a_{i}=1\right\} .
$$

(Again the conditions $\varphi_{i} \geq 0$ are only here to limit translations ensuring that $0 \in A$. This is essential in the numerical method.) These are very simple constraints on the admissible values, so step 3 in the algorithm is an elementary projection onto $\mathbb{R}_{+}^{n}$ and a hyperplane. Hence the given algorithm can be implemented in a straightforward way.

We present an example result on figure 3. Here we chose at random 999 vectors $\nu_{i}$ on $\mathbf{S}^{2}$, and 999 numbers $a_{i}$ in $[0,1]$ uniformly; $\nu_{1000}$ and $a_{1000}$ are determined such that the existence condition $\sum_{i=1}^{1000} a_{i} \nu_{i}=0$ is satisfied.

\subsection{Application: Cheeger sets}

Let us now present a more involved application. In 1970, Jeff Cheeger [11] proposed to study the problem

$$
\inf _{X \subset M} \frac{\mathcal{H}^{n-1}(\partial X)}{\mathcal{H}^{n}(X)}
$$


where $M$ is an $n$-dimensional manifold with boundary. The resulting optimal value, known as the Cheeger constant, can be used to give bounds for the first eigenvalue of the Laplace-Beltrami operator on $M$, and even more general operators [14]. There is a number of variations and applications of this problem, see for example $[2,16]$.

The theoretical results on the problem (6) are rather sparse. It is easy to show that the infimum is usually not attained in this general formulation. On the other hand it can be proved that minimizers exist whenever $M=\bar{\Omega}$, where $\Omega \subset \mathbb{R}^{n}$ is a nonempty open set. Moreover, if $\Omega$ is convex and $n=2$, there is a unique convex optimum $X$ which can be computed by algebraic algorithms [18]. On the other hand, if $n \geq 3$, it is not known whether the optimum set is unique or convex, even with $\Omega$ convex. However $\Omega$ convex implies that there exists at least one convex optimum [17]. But this optimum is not known for any particular $\Omega$ except balls.

Our algorithm allows us to compute an approximation of a convex optimum when $\Omega \subset \mathbb{R}^{3}$ is convex. Indeed (6) can be reformulated as follows:

$$
\min _{A \in \mathcal{A}} \frac{3 \int_{\partial A} d \mathcal{H}^{2}(x)}{\int_{\partial A} \varphi_{A}(x) d \mathcal{H}^{2}(x)}, \text { with } \mathcal{A}=\{A \subset \bar{\Omega}, A \text { convex and 3-dimensional }\}
$$

So the numerator and denominator here have the form $\int_{\partial A} f\left(\nu_{A}, \varphi_{A}\right)$, and our algorithm can be applied with straightforward modifications.

A key difference with respect to our previous application is the management of the constraint $A \subset \bar{\Omega}$. The set $\Omega$ itself is approximated by a polytope (whenever necessary). The corresponding enclosing half-spaces are kept in the algorithm in order to ensure that the approximating polytopes belong to $\mathcal{A}$. For example, if $\Omega$ is a unit cube, we fix $\nu_{1}=(1,0,0), \ldots, \nu_{6}=(0,0,-1)$ and $\varphi_{1}=\cdots=\varphi_{6}=1$.

This approach allows to handle any problem with constraints of the form

$$
Q_{0} \subset A \subset Q_{1}
$$

assuming that $Q_{1}$ is convex. (For $Q_{0}$ it is not a restriction to assume it is convex.) Other examples of problems of this kind come from mathematical economy, see references in [9], and also [4].

\section{Newton's problem of the body of minimal resistance}

The problem of the body of minimal resistance has been settled by I. Newton in its Principia: given a body progressing at constant speed in a fluid, what 


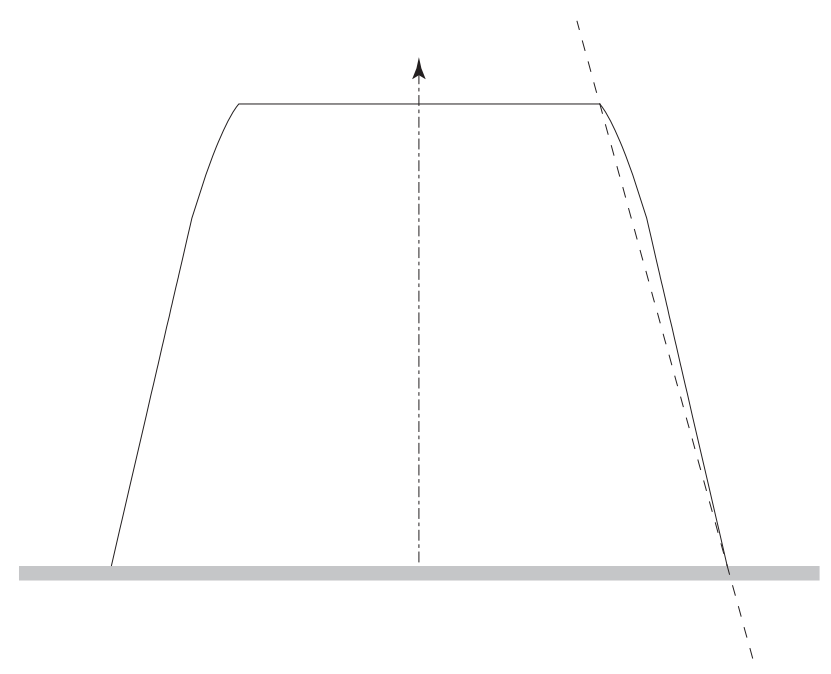

Figure 5: Profile of computed optimal shape $(M=3 / 2)$ : the solution is not developable.

shape should it be given in order to minimize its resistance? Expressed in its more classical way, this can be formulated as the following optimization problem:

$$
\min _{\substack{u: \Omega \rightarrow[-M, 0] \\ u \operatorname{convex}}} \int_{\Omega} \frac{d x}{1+|\nabla u|^{2}}
$$

where $M>0$ is a given parameter and $\Omega$ is the unit disk of $\mathbb{R}^{2}$. There is a lot of variants from this formulation and a huge literature on this problem, see $[5,19]$ and their references.

I. Newton considered only radial solutions of this problem, and his solution was already considered surprising. But it has been proved in [3] that the solutions of (8) are not radially symmetric. Unfortunately it has been impossible until now to describe more precisely the minimizers. Some theoretical results suggests that they should be developable in a sense given in [19]: a developable body being the convex hull of the unit circle in the plane $x_{3}=0$ and a convex set in the plane $x_{3}=-M$.

So in this application, we are considering a problem of the form (1), with $g(x, u, p)=1 /\left(1+|p|^{2}\right)$. As explained in Section 1.3, this can be reformulated as $(2)$ with $f(x, \nu, \varphi)=\left(\nu_{3}\right)_{+}^{3}$, where $t_{+}:=\max (t, 0)$ for any $t \in \mathbb{R}$. The set $\mathcal{A}$ is the set of convex bodies with a constraint of the kind (7), with $Q_{0}:=\Omega \times\{0\}$ and $Q_{1}:=\Omega \times[0, M]$.

In the classical application, $\Omega$ is a disk. So we discretize these constraints by replacing the disk by a regular polygon $\Omega_{\ell}$, with $\ell$ sides. (In practice we 


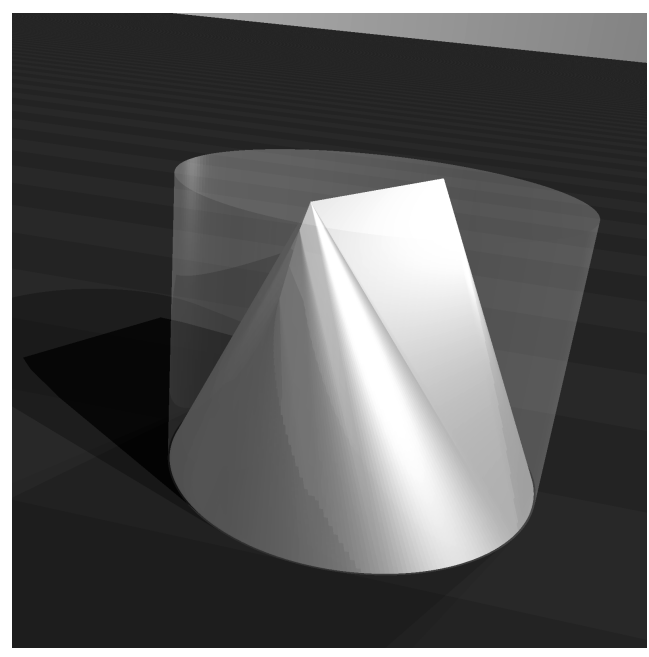

$M=3 / 2$

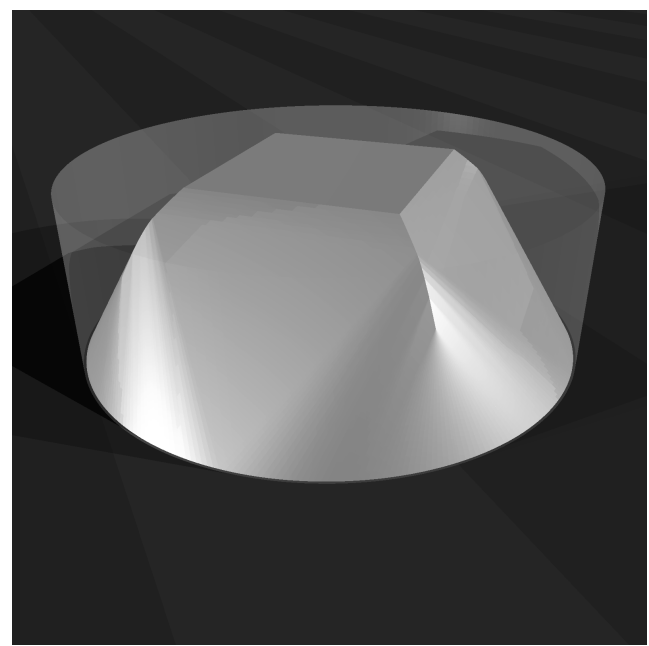

$M=7 / 10$

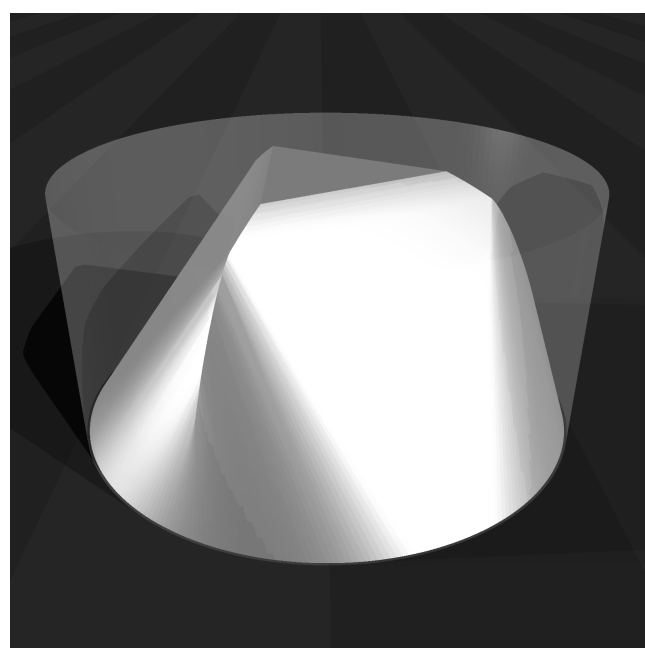

$M=1$

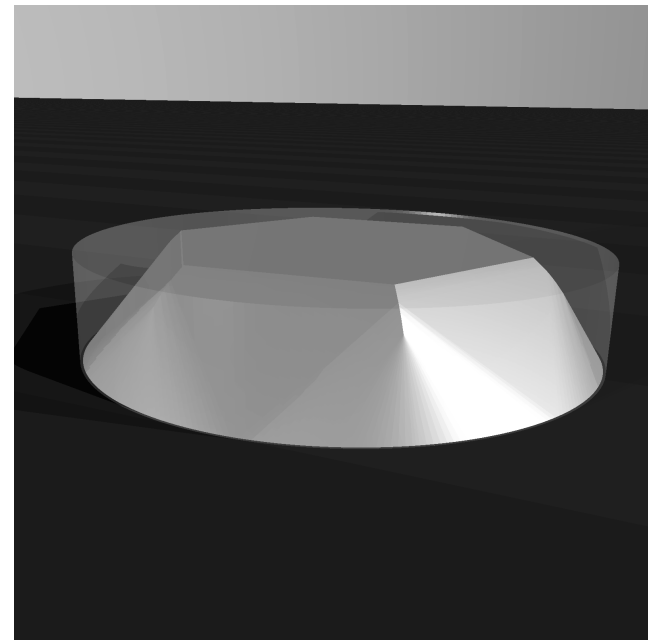

$M=4 / 10$

Figure 6: Computed solutions of Newton's problem of the body of minimal resistance.

used $\ell=300$.) In this particular problem, this yields an overestimated value of the functional. Indeed if $A \subset \Omega_{\ell} \times[0, M]$ is convex, then $\tilde{A}:=A \cap Q_{1}$ belongs to $\mathcal{A}$, and $\mathcal{F}(\tilde{A}) \leq \mathcal{F}(A)$ since $f \geq 0$ and vanishes on $\partial \tilde{A} \backslash \partial A$, where the normal vectors belong to $\left\{e_{3}\right\}^{\perp}$. Obviously for a minimization problem, this is not a predicament to overestimate the functional. 


\begin{tabular}{|c|c|c|c|}
$M$ & Newton's radial value & best theoretical values & numerical values \\
\hline $3 / 2$ & 0.7526 & 0.7019 & 0.7012 \\
1 & 1.1775 & 1.1561 & 1.1379 \\
$7 / 10$ & 1.5685 & 1.5566 & 1.5457 \\
$4 / 10$ & 2.1074 & 2.1034 & 2.1006
\end{tabular}

Table 1: Minimal values of the Newton's resistance.

Using our gradient method on this problem yields different results starting with different initial shapes. This is likely the consequence of the existence of local minima. (Note that no theoretical result is known on the number or on the kind of critical points in this problem.) So our method needs to be preprocessed to start closer from a global minimum.

We use a genetic algorithm for this task. It is inspired from the ideas developed by J. Holland [15].

Our tests exhibit a behavior corresponding to the theoretical results given in [19]. Even for local minimizers, the image set of $\nu_{A}$ is sparse in $\mathbf{S}^{2}$. This suggests that optimal sets could be described with a lot fewer parameters as convex hulls of points instead of as an intersection of half-spaces. Therefore, we use the information given in the stochastic step (from the genetic algorithm) in two ways: as an initial set for the gradient method, and as an initial guess of the appropriate set of normal vectors to use. But the stochastic step itself represents the convex bodies as convex hull of points in $\Omega_{\ell} \times[0, M]$, together with the vertices $\Omega_{\ell} \times\{0\}$. The genetic algorithm optimizes the position of these points.

With these improvements, we get similar shapes for any run of the algorithm. Some of them are pictured in Figure 3, for different values of the parameter $M$. These solutions are not developable in the sense of [19]. This can be seen more precisely on Figure 5, where only the profile of the body is pictured.

Note that the corresponding values obtained by our method are smaller than the best theoretical values described in [19], even though they are slightly overestimated as explained before: see Table 1.

It is a common conjecture on this problem that the solution is smooth except on the top and bottom parts, that is on $u^{(-1)}(0, M)$. However $C^{2}$ regularity would imply the developability property [19, Conjecture 2]. Our results demonstrate the non optimality of the best previously known profiles, and consequently the non regularity of the minimizers. 


\section{References}

[1] A. D. Alexandrov, Theory of mixed volumes for convex bodies, Mathem. Sb. USSR 2 (1937), pp. 947-972.

[2] G. Bellettini, V. Caselles \& M. Novaga, The total variation flow in $\mathbb{R}^{N}$, J. Differential Equations 184 (2002), pp. 475-525.

[3] F. Brock, V. Ferone, B. Kawohl, A Symmetry Problem in the Calculus of Variations, Calc. Var. Partial Differential Equations, 4 (1996), pp. 593599.

[4] G. Buttazzo, P. Guasoni, Shape optimization problems over classes of convex domains, J. Convex Anal. 4 (1997), no. 2, pp. 343-351.

[5] G. Buttazzo, V. Ferone \& B. Kawohl, Minimum Problems over Sets of Concave Functions and Related Questions, Math. Nachrichten, 173 (1993), pp. 71-89.

[6] G. Carlier, On a theorem of Alexandrov, to appear.

[7] G. Carlier \& T. Lachand-Robert, Convex bodies of optimal shape. J. Convex Anal. 10 (2003) pp. 265-273.

[8] G. Carlier \& T. Lachand-Robert, Regularity of solutions for some variational problems subject to convexity constraint, Comm. Pure Appl. Math. 54 (2001), pp. 583-594.

[9] G. Carlier, T. Lachand-Robert \& B. Maury, $H^{1}$-projection into sets of convex functions: a saddle point formulation, Proceedings ESAIM (2001).

[10] G. Carlier, T. Lachand-Robert \& B. Maury, A numerical approach to variational problems subject to convexity constraint, Numerische Math. 88 (2001), pp. 299-318.

[11] Cheeger, J., A lower bound for the smalles eigenvalue of the Laplacian, in: Problems in Analysis, A Symposium in Honor of Salomon Bochner, Ed.: R.C.Gunning, Princeton Univ. Press (1970) pp. 195-199.

[12] P. Choné \& J.-C. Rochet, Ironing, Sweeping and Multidimensional screening, Econometrica, vol. 66 (1998), pp. 783-826. 
[13] P. Choné \& H. Le Meur, Non-convergence result for conformal approximation of variational problems subject to a convexity constraint, Numer. Funct. Anal. Optim. 22 (2001), no. 5-6, pp. 529-547.

[14] V. Fridman \& B. Kawohl, Isoperimetric estimates for the first eigenvalue of the p-Laplace operator and the Cheeger constant, Comment. Math. Univ. Carol. 44 (2003) pp. 659-667.

[15] J. Holland, Adaptation in natural and artificial systems, Univ. Michigan Press (1975).

[16] I. Ionescu \& T. Lachand-Robert, Generalized Cheeger sets related to landslides, submitted.

[17] B. Kawohl, On a family of torsional creep problems, J. reine angew. Math. 410 (1990) pp. 1-22.

[18] B. Kawohl \& T. Lachand-Robert, Characterization of Cheeger sets for convex subsets of the plane, to appear.

[19] T. Lachand-Robert \& M. A. Peletier, Newton's problem of the body of minimal resistance in the class of convex developable functions, Math. Nachrichten 226 (2001), pp. 153-176.

[20] P.-L. Lions, Identification du cône dual des fonctions convexes et applications, C. R. Acad. Sci. Paris (1998).

[21] H. Minkowski, Allgemeine Lehrsätze über die Konvexen Polyeder, Nach. Ges. Wiss., Göttingen (1897), pp. 198-219.

[22] R. T. Rockafellar, Convex Analysis, Princeton University Press (1970). 